\title{
The Effect of Algae or Insect Supplementation as Alternative Protein Sources on the Volatile Profile of Chicken Meat
}

 \\ Daniel Mörlein ${ }^{1}$ (D)
}

1 Department of Animal Sciences, University of Göttingen, Kellnerweg 6, 37077 Göttingen, Germany; marco.ciulu@uni-goettingen.de (M.C.); brianne.altmann@agr.uni-goettingen.de (B.A.A.); daniel.moerlein@uni-goettingen.de (D.M.)

2 Breeding Informatics Group, Department of Animal Sciences, Georg-August University, Margarethe von Wrangell-Weg 7, 37075 Göttingen, Germany; armin.schmitt@uni-goettingen.de

3 Center for Integrated Breeding Research (CiBreed), University of Göttingen, Albrecht-Thaer-Weg 3, 37075 Göttingen, Germany

* Correspondence: vasiliki.gkarane@uni-goettingen.de or v.gkarane@qub.ac.uk

Received: 17 July 2020; Accepted: 1 September 2020; Published: 4 September 2020

\begin{abstract}
The aim of this study was to investigate the differences in the volatile profile of meat from chickens fed with alternative protein diets (such as algae or insect) through two different trials. In Trial 1, broiler chicken at one day of age were randomly allocated to three experimental groups: a basal control diet (C) and two groups in which the soybean meal was replaced at 75\% (in the starter phase) and 50\% (in the grower phase) with partially defatted Hermetia illucens (HI) larvae or Arthrospira platensis (SP). In Trial 2, broiler chickens were housed and reared similar to Trial 1, with the exception that the experimental diets replaced soybean meal with either 100\% partially defatted $\mathrm{HI}$ or 100\% SP. In both trials, chickens were slaughtered at day 35. Per group, 10 chickens were submitted to volatile analysis by using solid-phase microextraction (HS-SPME) and gas chromatography-mass spectrometry (GC-MS) analysis. Results in both trials showed that levels of several lipid-derived compounds were found to be lower in chickens fed an HI diet, which could be linked to a possibly lower level of polyunsaturated fatty acid content in HI-fed chicken. In addition, the dietary treatments could be discriminated based on the volatile profile, i.e., the substitution of soy with HI or SP distinctively affected the levels of flavor compounds.
\end{abstract}

Keywords: volatile compounds; sustainable feeds; spirulina; Arthrospira platensis; insect; Hermetia illucens

\section{Introduction}

Intake of high-quality protein, in the range of 1.2-1.6 g per $\mathrm{kg}$ body weight per day, has been associated with health benefits, with poultry identified as one of the main sources [1]. Chicken meat consumption is popular for a number of reasons, not least of which are its nutritional value (i.e., the lower content of saturated fat and the higher proportion of polyunsaturated fatty acids (PUFA) compared to other types of meat), its affordable price, and its lack of religious and cultural constraints associated with its consumption [2].

Chicken meat composition and quality are highly associated with animal diet [3], which once supplemented with functional ingredients (e.g., antioxidants, n-3 PUFA) may enhance the nutritional value of meat [4]. To date, corn, soybean, and fishmeal are the main conventional components of poultry diets [5]. However, despite the high protein content and the well balanced amino acid profile that these ingredients provide [6,7], they are often linked with a negative environmental footprint (i.e., land 
degradation, water deprivation, greenhouse gas emissions, or marine overexploitation) [8]. In light of the forthcoming increase of the global population (expected to reach nine billion by 2050) [9], the rising consumer demand for high quality protein, along with the on-going environmental deterioration and climate change, there is an urgent need to switch to more sustainable feeds in animal production systems $[10,11]$.

The use of microalga and insects have gained increasing attention in the last 30 years, holding the potential to act as alternative economical and more sustainable protein source for the livestock sector $[12,13]$. One of the most widely studied insect species is the black soldier fly (Hermetia illucens; $\mathrm{HI}$ ), the larvae of which contains high levels of protein (approximately $45 \%$ dry matter (DM)) $[14,15]$ and has an optimal amino acid composition [16]. In addition, studies in poultry meat show that growth performance, carcass traits, feeding efficiency $[17,18]$, and several meat quality traits $(\mathrm{pH}$, meat color, drip loss, and proximate or sensory analysis parameters) [18-20] are not negatively affected when soybean meal is partially or totally replaced by HI larvae.

Arthrospira platensis (commonly known as spirulina (SP)) is an edible microalga species with high nutritional value characterized by a $55-70 \%$ protein content [21]. Moreover, it has $\gamma$-linolenic acid, n-3 long chain, essential amino acids, vitamins, minerals, antioxidants, and carotenoids [22]. Khan et al. [23] summarizes the information available on the rich biological activity of spirulina including its antimicrobial, antioxidant, antiviral, anti-inflammatory, and immune-modulating role. Inclusion of microalgae in the poultry diet can affect the performance, oxidative stability, and meat quality parameters of broiler chickens [24-26].

Animal feeding strategies can modify the fatty acid profile of meat, as reflected in its volatile profile [27]. These interventions can influence the nutritional value, the eating quality, and consumer acceptance of meat [28]. Volatile compounds, formed mainly through after meat is cooked [29], have been widely used in meat provenance investigation studies as a way to trace, identify, or discriminate animal feeding systems [27,30]. Interestingly, differences in the volatile profile of chicken meat deriving from conventional vs. the aforementioned alternative protein sources have not yet been reported. Thus, the aim of this study was to investigate the volatile profile of chicken breast meat produced with a traditional soybean meal based diet (control, $\mathrm{C}$ diet) or diets with a partial substitution ( $75-50 \%$ in Trial 1) and complete substitution (100\% in Trial 2) of soybean meal with either defatted HI or SP.

\section{Materials and Methods}

\subsection{Birds and Diet}

The studies were carried out at the Department of Animal Sciences, University of Göttingen, and approved by the Ethics Committee of the Lower Saxony Office for Consumer Protection and Food Safety (LAVES; \#33.9-42502-04-15/2027), Germany. The trials were part of a larger animal nutrition study, which investigated the effects of both alternative protein feeds on performance and apparent digestibility [31]. The trials were analyzed separately, as they were not exact replicates (i.e., substitution levels, spirulina source, sampling time, and storage temperature differed among the two trials). Ross-308 male broiler chickens were slaughtered at 35 days of age at the University of Göttingen poultry slaughterhouse, which is regulated by article 4 of the European Union's (EG) NR. 853/2004 37. Immediately following slaughter, the carcasses were weighed and butchered, where the breasts (m. pectoralis major) were skinned and cooled to $4{ }^{\circ} \mathrm{C}(5 \mathrm{~h})$, and then later frozen until further analysis. Average carcass weighed $1.77 \pm 0.30 \mathrm{~kg}$ and $1.75 \pm 0.24 \mathrm{~kg}$ for Trial 1 and Trial 2, respectively.

\subsection{Trial 1 ( $75 \%$ to $50 \%$ Replacement)}

The experiment was divided into a starter feeding phase (1-21 days) and a grower feeding phase (22-34 days). One-day-old chicks were randomly allocated to floor pens with 5.8 birds $\mathrm{m}^{-2}$ (i.e., 7 birds per pen). Average body weights per pen were similar at the start of the study. Feed and water were 
available ad libitum. The control diet (C) was based on wheat, corn, and soybean meal as the main ingredients. The experimental diets replaced soybean meal with either $75 \%$ partially defatted $\mathrm{HI}$ or $\mathrm{SP}$ in the starter phase and either $50 \% \mathrm{HI}$ or SP in the grower phase. HI meal was sourced from a commercial producer (Hermetia Baruth $\mathrm{GmbH}$, Baruth/Mark, Germany). The larvae were fattened on a rye and wheat bran substrate, dried at $65-70{ }^{\circ} \mathrm{C}$, and partially-defatted with a screw press, then ground into a meal until an ultimate crude protein content $60.8 \%$ of DM and crude lipid content $14.1 \%$ of DM. Spirulina were sourced commercially from Myanmar, harvested, and sun-dried prior to packaging (crude protein content $58.8 \%$ of DM and crude lipid content $4.3 \%$ of DM). Amino acids were supplemented according to breeder guidelines. A total of 30 chicken breast samples were analyzed $(10 \mathrm{C}, 10 \mathrm{HI}$, and $10 \mathrm{SP})$. Samples originated from the chicken breast (m. pectoralis major) and were vacuum-packed in polyamide/polyethylene (PA/PE) bags and stored at $-72{ }^{\circ} \mathrm{C}$ for 31 months until analysis. Full details of the animal and production characteristics and composition of feeds were described by Neumann et al. [31] (experiment 2).

\subsection{Trial $2(100 \%$ Replacement)}

This experiment was also divided into a starter (1-21 days) and a grower feeding phase (22-34 days), and chicks were housed and reared as in Trial 1 . One major modification is that the experimental diets replaced soybean meal with either $100 \%$ partially defatted $\mathrm{HI}$ or $100 \%$ SP in both feeding phases. $\mathrm{HI}$ meal was produced by a commercial producer (Hermetia Baruth $\mathrm{GmbH}$, Baruth/Mark, Germany) with an ultimate crude protein content $60.8 \%$ of DM and crude lipid content $14.1 \%$ of DM. Spirulina were sourced commercially from China; the product was harvested, rinsed, and spray-dried prior to packaging (crude protein content $68.9 \%$ of DM and crude lipid content $6.3 \%$ of DM). A total of 30 chicken breast samples (10 C, $10 \mathrm{HI}$ and $10 \mathrm{SP}$ ) were analyzed. Samples (m. pectoralis major) were vacuum-packed in PA/PE bags and stored at $-20{ }^{\circ} \mathrm{C}$ for 26 months until analysis. Full details of the animal and production characteristics and composition of feeds were described by Neumann et al. [31] (experiment 3).

\subsection{Sample Preparation and Volatile Compound Analysis}

Volatile compounds were analyzed using headspace solid phase microextraction (HS-SPME). Before use, chicken breasts were thawed at room temperature by immersion of the frozen vacuum-packed samples in water, for $10 \mathrm{~min}$. White tendons were removed and samples were minced by means of a mini chopper (DS Produkte GmbH, Gallin, Germany). Next, $4.5 \pm 0.05 \mathrm{~g}$ of meat were weighed and transferred into $20 \mathrm{~mL}$ glass headspace vials together with $5 \mu \mathrm{L}$ of a methanolic solution of 5 ppm 1,2-dichlorobenzene (Sigma-Aldrich, Munich, Germany), as internal standard. Vials were sealed with polytetrafluoroethylene (PTFE)-faced silicone septum (Macherey-Nagel, Düren, Germany). Meat samples were heated at $70{ }^{\circ} \mathrm{C}$ for $10 \mathrm{~min}$ in a TriPlus $\mathrm{RSH}^{\mathrm{TM}}$ autosampler (Thermo Fisher Scientific, Waltham, MA, USA) for equilibration, before exposing the 30/50 $\mu \mathrm{m}$ Divinylbenzene/Carboxen/Polydimethylsiloxane (DVB/CAR/PDMS) fiber (Supelco, Bellefonte, PA, USA) into the headspace, where it was held for $20 \mathrm{~min}$ under constant stirring. Before its first use, the solid phase microextraction (SPME) fiber was thermally pre-conditioned at $270{ }^{\circ} \mathrm{C}$ for $1 \mathrm{~h}$ in accordance with the manufacturer recommendation.

\subsection{Gas Chromatography-Mass Spectrometry (GC-MS) Analyses}

The GC-MS analyses of the samples were performed using a TRACE ${ }^{\mathrm{TM}} 1310$ gas-chromatograph coupled with an ISQ-LT single quadrupole mass spectrometer (Thermo Fisher Scientific, Waltham, MA, USA). The SPME fiber was thermally desorbed in a programmed temperature vaporizing injector at $250{ }^{\circ} \mathrm{C}$ in a splitless mode for $2 \mathrm{~min}$ while a split ratio of 1:20 was adopted for the remaining time of the chromatographic run. Inlet temperature was set at $250{ }^{\circ} \mathrm{C}$ while a desorption time of $7 \mathrm{~min}$ was adopted. Separation of compounds was achieved by a TG-5SILMS column $(30 \mathrm{~m} \times 0.25 \mathrm{~mm} \times 0.25 \mu \mathrm{m})$ provided by Thermo Scientific. Helium was used as carrier gas operating at $1 \mathrm{~mL} \mathrm{~min}{ }^{-1}$. Oven temperature was 
kept at $40{ }^{\circ} \mathrm{C}$ during the first $5 \mathrm{~min}$, increased to $250{ }^{\circ} \mathrm{C}$ at $4{ }^{\circ} \mathrm{C} \min ^{-1}$, and was maintained at $250{ }^{\circ} \mathrm{C}$ during the last $2 \mathrm{~min}$. After desorption, a fiber bake out was carried out in a bake out unit for $20 \mathrm{~min}$ at $260{ }^{\circ} \mathrm{C}$ to avoid carry-over phenomena among subsequent samples. The total chromatographic run time was $59.5 \mathrm{~min}$. The MS detector operated in ion scan mode (45-230 amu), ion source, and transfer line temperature were maintained at $250{ }^{\circ} \mathrm{C}$ and $270{ }^{\circ} \mathrm{C}$, respectively. The electron energy was $70 \mathrm{eV}$. Identification of the compounds was performed comparing (a) experimental mass spectra to those contained in the National Institute of Standards and Technology database (NIST/EPA/NIH Mass Spectral Library, Version 2.2, 2014) and (b) linear retention indexes (LRIs) based on a homologous series of n-alkanes (C7-C30, Sigma-Aldrich, Munich, Germany) with those reported in other studies. Data were expressed in ng per g meat using the formula provided by Wang et al. [32].

\subsection{Data Analysis}

GC-MS data were aligned per trial based on retention time in the R Core Team [33] using the package 'CGalignR' [34]. For a better match of slightly shifted peaks, a full join function provided by the package 'fuzzyjoin' [35] was applied, to allow for peak alignment until a maximum difference of 0.03 was achieved in both directions.

Volatile data did not meet the assumption of normal distribution. For this reason, the nonparametric Kruskal-Wallis test was applied in order to test the effect of dietary treatment on the volatile profile of chicken meat in each trial. In the case of a significant result of the Kruskal-Wallis test, the Wilcoxon rank-sum-test was applied to compare the medians of two groups. Differences were considered to be significant when $p<0.05$. The results are presented as medians. Median absolute deviation was reported as measure of dispersion, defined as the median [absolute $\left(x_{i}-\right.$ median $\left.(x)\right)$ ], where $x_{i}$ is an individual observation and median $(x)$ is the median of values $x$. Linear discriminant analysis (LDA) was applied to investigate the potential separation of the dietary treatments within each trial [36] and to detect variables (compounds) that were more discriminant among feeding treatments. In order to detect the discriminatory efficiency of LDA, a confusion matrix followed by Cohen's Kappa coefficient value was computed [37]. An individual animal was considered as the experimental unit.

\section{Results}

In total, 61 compounds in Trial 1 and 65 compounds in Trial 2, appearing in at least $60 \%$ of the samples (i.e., in 18 or more out of the 30 samples in each trial) were tentatively identified (Supplementary Table S1). The compounds that showed significant differences in the medians in Kruskal-Wallis test and the compounds constituting the minimal discriminatory set are marked in Table 1 (for Trial 1) and Table 2 (for Trial 2). Four compounds in each trial were not identified.

Table 1. The composition of the volatile profile of chicken breast meat fed with three different diets in Trial 1 ((Control (C) vs. Spirulina (SP) vs. Hermetia illucens (HI)) (results expressed as ng/g).

\begin{tabular}{|c|c|c|c|c|c|}
\hline & $C^{1}$ & $\mathrm{SP}^{1}$ & $\mathrm{HI}^{1}$ & $\begin{array}{c}\text { Statistical } \\
\text { Analysis }\end{array}$ & $p$-Level \\
\hline \multicolumn{6}{|l|}{ Alcohols } \\
\hline 2-methoxy-ethanol & $9.36(13.17)$ & $9.50(5.57)$ & $8.45(3.60)$ & & \\
\hline 1-Penten-3-ol & $4.48(9.87)$ & $6.15(5.33)$ & $7.86(4.12)$ & & \\
\hline 1-Pentanol & $2.13^{\mathrm{a}}(3.17)$ & $2.54^{\mathrm{a}}(1.95)$ & $0.11^{\mathrm{b}}(0.11)$ & KW; LDA & 0.026 \\
\hline 1-Hexanol & $1.26^{\mathrm{ab}}(1.57)$ & $1.40^{\mathrm{a}}(0.59)$ & $0.71^{\mathrm{b}}(0.23)$ & KW; LDA & 0.030 \\
\hline 1-Heptanol & $0.87^{\mathrm{a}}(0.92)$ & $0.73^{\mathrm{a}}(0.31)$ & $0.32^{\mathrm{b}}(0.08)$ & KW; LDA & 0.027 \\
\hline 1-Octen-3-ol & $21.3^{\mathrm{ab}}(21.6)$ & $31.4^{\mathrm{a}}(10.1)$ & $13.2^{\mathrm{b}}(1.77)$ & KW; LDA & 0.034 \\
\hline 2-Ethyl-2-hexenol & $4.02(4.13)$ & $2.17(0.86)$ & $1.57(0.85)$ & & \\
\hline 4-Ethyl-cyclohexanol & $0.00(0.53)$ & $0.00(0.00)$ & $0.00(0.00)$ & & \\
\hline 2-Ethyl-1-hexanol & $4.70(6.76)$ & $5.26(3.16)$ & $4.41(3.15)$ & & \\
\hline 2-Ethyl-1-decanol & $1.11(1.11)$ & $0.77(0.40)$ & $0.51(0.21)$ & & \\
\hline 2-Octen-1-ol & $0.75(0.77)$ & $0.88(0.47)$ & $0.34(0.24)$ & & \\
\hline 1-Octanol & $4.02(4.25)$ & $3.19(0.88)$ & $2.91(0.77)$ & & \\
\hline Benzenemethanol, $\alpha, \alpha$-dimethyl & $0.61(0.78)$ & $0.46(0.34)$ & $0.56(0.51)$ & & \\
\hline (Z)-2-Nonen-1-ol & $1.14(1.01)$ & $0.79(0.38)$ & $0.54(0.20)$ & & \\
\hline 1-Nonanol & $0.21(0.25)$ & $0.25(0.07)$ & $0.26(0.16)$ & & \\
\hline
\end{tabular}


Table 1. Cont.

\begin{tabular}{|c|c|c|c|c|c|}
\hline & $C^{1}$ & $\mathrm{SP}^{1}$ & HI $^{1}$ & $\begin{array}{c}\text { Statistical } \\
\text { Analysis }\end{array}$ & $p$-Level \\
\hline \multicolumn{6}{|l|}{ Aldehydes } \\
\hline Pentanal & $4.16(7.53)$ & $7.08(4.95)$ & $3.52(3.52)$ & & \\
\hline Hexanal & $148.1^{\mathrm{ab}}(143.4)$ & $164.3^{\mathrm{a}}(42.8)$ & $78.62^{\mathrm{b}}(41.7)$ & KW; LDA & 0.043 \\
\hline Heptanal & $3.48(4.33)$ & $4.89(1.46)$ & $2.94(1.13)$ & & \\
\hline Methional & nd & nd & nd & & \\
\hline 2-Heptenal & $0.52^{\mathrm{ab}}(1.12)$ & $0.75^{\mathrm{a}}(0.45)$ & $0.13^{\mathrm{b}}(0.13)$ & KW; LDA & 0.022 \\
\hline Benzaldehyde & $0.49(0.66)$ & $0.00(0.00)$ & $1.0(0.80)$ & & \\
\hline Octanal & $7.91(8.01)$ & $7.74(3.00)$ & $5.98(1.07)$ & & \\
\hline 2-Octenal & $0.84(1.09)$ & $1.01(0.45)$ & $0.70(0.37)$ & & \\
\hline Nonanal & $23.8(18.2)$ & $23.7(10.1)$ & $17.5(5.73)$ & & \\
\hline (E)-2-Nonenal & $0.30(0.32)$ & $0.38(0.14)$ & $0.24(0.05)$ & & \\
\hline (Z)-4-Decenal & $0.00^{a}(0.00)$ & $0.00^{\mathrm{a}}(0.00)$ & $0.00^{\mathrm{b}}(0.00)$ & KW; LDA & 0.026 \\
\hline Decanal & $0.96(1.37)$ & $1.37(0.74)$ & $0.89(0.49)$ & & \\
\hline 2-Decenal & $0.20(0.22)$ & $0.29(0.13)$ & $0.15(0.05)$ & & \\
\hline$(E, E)$-2,4-Decadienal & $0.00^{\mathrm{b}}(0.18)$ & $0.17^{\mathrm{a}}(0.11)$ & $0.06^{\mathrm{ab}}(0.06)$ & KW; LDA & 0.028 \\
\hline (E)-2-Undecenal & $0.10(0.12)$ & $0.12(0.05)$ & $0.06(0.02)$ & & \\
\hline Dodecanal & $0.24(0.24)$ & $0.30(0.12)$ & $0.20(0.06)$ & & \\
\hline Tridecanal & $0.04^{\mathrm{ab}}(0.05)$ & $0.07^{\mathrm{a}}(0.04)$ & $0.01^{\mathrm{b}}(0.01)$ & KW; LDA & 0.029 \\
\hline Tetradecanal & $0.06^{\mathrm{ab}}(0.06)$ & $0.08^{a}(0.03)$ & $0.03^{\mathrm{b}}(0.03)$ & KW; LDA & 0.031 \\
\hline \multicolumn{6}{|l|}{ Ketones } \\
\hline 2-Heptanone & $0.79(1.03)$ & $0.81(0.34)$ & $0.83(0.23)$ & & \\
\hline Butyrolactone & nd & nd & nd & & \\
\hline 2-Methyl-6-heptanone & $0.13(0.55)$ & $0.03(0.03)$ & $0.00(0.00)$ & & \\
\hline 2-Nonanone & $0.08^{\mathrm{ab}}(0.12)$ & $0.00^{\mathrm{b}}(0.00)$ & $0.38^{\mathrm{a}}(0.32)$ & KW; LDA & 0.005 \\
\hline \multicolumn{6}{|l|}{ Hydrocarbons } \\
\hline Toluene & nd & nd & nd & & \\
\hline 1,2,4-Trimethyl-cyclopentane & nd & nd & nd & & \\
\hline Propyl-cyclohexane & $0.00(2.16)$ & $0.00(0.00)$ & $0.00(0.00)$ & & \\
\hline 4-Methyl-nonane & nd & nd & nd & & \\
\hline 2,2,6-Trimethyl-octane & nd & nd & nd & & \\
\hline 2,2,4,6-Pentamethyl-heptane & $40.4(33.5)$ & $39.7(16.0)$ & $27.2(8.66)$ & & \\
\hline Decane & $0.41(0.42)$ & $0.29(0.24)$ & $0.18(0.14)$ & & \\
\hline 2,2,4,4-Tetramethyl-octane & $3.16(3.42)$ & $2.40(1.05)$ & $2.23(1.06)$ & & \\
\hline 2,6,7-Trimethyl-decane & $0.67(0.63)$ & $0.51(0.35)$ & $0.34(0.34)$ & & \\
\hline 2-Methyl-decane & $0.00(0.09)$ & $0.00(0.00)$ & $0.00(0.00)$ & & \\
\hline 5-Undecene & nd & nd & nd & & \\
\hline Undecane & $1.10(1.18)$ & $1.09(0.21)$ & $1.02(0.42)$ & & \\
\hline 2,8-Dimethyl-4-methylene-nonane & nd & nd & nd & & \\
\hline Pentyl-cyclohexane & nd & nd & nd & & \\
\hline 3-Methylene-undecane & $0.00(0.07)$ & $0.00(0.00)$ & $0.00(0.00)$ & & \\
\hline Dodecane & $1.16(1.23)$ & $1.22(0.45)$ & $0.95(0.39)$ & & \\
\hline 2,6,11-Trimethyl-dodecane & $0.17(0.19)$ & $0.30(0.10)$ & $0.23(0.09)$ & & \\
\hline Tridecane & $0.55(0.62)$ & $0.79(0.35)$ & $0.58(0.20)$ & & \\
\hline 2,3,5,8-Tetramethyl-decane & $0.19(0.19)$ & $0.23(0.08)$ & $0.20(0.06)$ & & \\
\hline Tetradecane & $0.52(0.50)$ & $0.61(0.22)$ & $0.48(0.14)$ & & \\
\hline Pentadecane & $0.36^{\mathrm{b}}(0.66)$ & $0.57^{\mathrm{ab}}(0.13)$ & $1.18^{\mathrm{a}}(0.75)$ & KW; LDA & 0.013 \\
\hline 2,6,10-Trimethyl-tetradecane & $0.07(0.08)$ & $0.10(0.03)$ & $0.08(0.05)$ & & \\
\hline Hexadecane & $0.21(0.21)$ & $0.28(0.06)$ & $0.20(0.06)$ & & \\
\hline Heptadecane & $0.03^{\mathrm{b}}(0.03)$ & $0.28^{\mathrm{a}}(0.10)$ & $0.00^{\mathrm{b}}(0.00)$ & KW; LDA & 0.001 \\
\hline \multicolumn{6}{|l|}{ Thiols } \\
\hline 2-Ethyl-1-hexanethiol & nd & nd & nd & & \\
\hline 2-Methyl-2-heptanethiol & nd & nd & nd & & \\
\hline \multicolumn{6}{|l|}{ Esters } \\
\hline $\begin{array}{c}\text { Pentanoic acid,2,2,4-trimethyl-3-hydroxy-, } \\
\text { isobutyl ester }\end{array}$ & $0.06(0.09)$ & $0.08(0.08)$ & $0.15(0.09)$ & & \\
\hline Carbamodithioic acid, diethyl-, methyl ester & $0.24(0.27)$ & $0.35(0.14)$ & $0.14(0.14)$ & & \\
\hline Dimethyl phtalate & $4.42(4.74)$ & $5.73(1.43)$ & $4.70(0.92)$ & & \\
\hline $\begin{array}{l}\text { Pentanoic acid, } \\
\text { 2,2,4-trimethyl-3-carboxyisopropy, } \\
\text { isobutyl ester }\end{array}$ & $0.00(0.01)$ & $0.00(0.00)$ & $0.00(0.00)$ & & \\
\hline \multicolumn{6}{|l|}{ Lactone } \\
\hline$\gamma$-nonalactone & $0.12^{\mathrm{b}}(0.12)$ & $0.20^{\mathrm{a}}(0.07)$ & $0.09^{\mathrm{b}}(0.03)$ & KW; LDA & 0.046 \\
\hline \multicolumn{6}{|l|}{ Acid } \\
\hline Dodecanoic acid & $0.00(0.01)$ & $0.00(0.02)$ & $0.00(0.00)$ & & \\
\hline \multicolumn{6}{|l|}{ Nitrile } \\
\hline 4-Cyano-cyclohexene & $0.00(0.23)$ & $0.00(0.00)$ & $0.16(0.16)$ & & \\
\hline
\end{tabular}


Table 1. Cont.

\begin{tabular}{|c|c|c|c|c|c|}
\hline & $C^{1}$ & $\mathrm{SP}^{1}$ & $\mathrm{HI}^{1}$ & $\begin{array}{l}\text { Statistical } \\
\text { Analysis }\end{array}$ & $p$-Level \\
\hline \multicolumn{6}{|l|}{ Azide } \\
\hline 2-Azido-2,4,4,6,6-pentamethyl-heptane & $0.15(0.26)$ & $0.05(0.05)$ & $0.15(0.11)$ & & \\
\hline \multicolumn{6}{|l|}{ Unknown } \\
\hline Unknown (RT:17.96 min) & $0.00^{\mathrm{b}}(0.00)$ & $0.00^{\mathrm{b}}(0.00)$ & $0.00^{\mathrm{a}}(0.00)$ & KW; LDA & 0.040 \\
\hline Unknown (RT:23.76 min) & $0.01(0.03)$ & $0.00(0.00)$ & $0.09(0.07)$ & LDA & \\
\hline Unknown (RT:28.62 min) & $0.00^{\mathrm{a}}(0.00)$ & $0.00^{\mathrm{b}}(0.00)$ & $0.00^{\mathrm{a}}(0.00)$ & KW; LDA & 0.012 \\
\hline Unknown (RT:37.40 min) & $0.00(0.02)$ & $0.00(0.00)$ & $0.00(0.00)$ & LDA & \\
\hline
\end{tabular}

1 , Values are expressed as: Median (Median absolute deviation); ${ }^{\mathrm{a}, \mathrm{b}}$, medians assigned different superscripts indicate significant differences $(p<0.05)$ between the dietary treatments; KW: Compounds that were found to be significantly different $(p<0.05)$ due to different dietary treatment, following the Kruskal-Wallis Test; LDA: Compounds that belong to the minimal set of 18 compounds that lead to a complete separation of the three dietary groups in linear discriminant analysis, i.e., to a confusion matrix with kappa $=1$; nd: not detected.

Table 2. The composition of the volatile profile of chicken breast meat fed with three different diets in Trial 2 ((Control (C) vs. Spirulina (SP) vs. Hermetia illucens (HI)) (results expressed as ng/g).

\begin{tabular}{|c|c|c|c|c|c|}
\hline & $C^{1}$ & $\mathrm{SP}^{1}$ & $\mathrm{HI}^{1}$ & $\begin{array}{l}\text { Statistical } \\
\text { Analysis }\end{array}$ & $p$-Level \\
\hline \multicolumn{6}{|l|}{ Alcohols } \\
\hline 2-Methoxy-ethanol & $3.43(2.11)$ & $1.62(1.58)$ & $1.71(1.10)$ & & \\
\hline 1-Penten-3-ol & $3.98(2.84)$ & $2.93(1.30)$ & $2.58(2.49)$ & & \\
\hline 1-Pentanol & $8.88^{\mathrm{a}}(5.76)$ & $9.89^{\mathrm{a}}(3.72)$ & $2.02^{\mathrm{b}}(1.08)$ & KW & 0.016 \\
\hline 1-Hexanol & $4.20^{\mathrm{a}}(2.59)$ & $4.69^{\mathrm{a}}(2.72)$ & $1.53^{b}(0.38)$ & KW; LDA & 0.003 \\
\hline 1-Heptanol & $1.44(0.83)$ & $2.49(0.86)$ & $1.52(0.49)$ & & \\
\hline 1-Octen-3-ol & $30.8^{a}(13.7)$ & $36.0^{\mathrm{a}}(9.44)$ & $11.6^{\mathrm{b}}(0.93)$ & KW; LDA & 0.001 \\
\hline 2-Ethyl-2-hexenol & nd & nd & nd & & \\
\hline 4-Ethyl-cyclohexanol & $4.39^{\mathrm{ab}}(4.39)$ & $6.30^{\mathrm{a}}(1.65)$ & $2.53^{b}(0.60)$ & KW & 0.009 \\
\hline 2-Ethyl-1-hexanol & $12.2(3.02)$ & $19.4(4.95)$ & $20.3(2.75)$ & & \\
\hline 2-Ethyl-1-decanol & $3.38(1.66)$ & $3.90(1.99)$ & $4.78(1.32)$ & & \\
\hline 2-Octen-1-ol & $0.32(0.32)$ & $1.34(1.34)$ & $1.53(0.73)$ & & \\
\hline 1-Octanol & $4.05^{\mathrm{ab}}(2.02)$ & $4.83^{\mathrm{a}}(1.15)$ & $2.61^{\mathrm{b}}(0.71)$ & KW & 0.045 \\
\hline Benzenemethanol, $\alpha, \alpha$-dimethyl & $0.21(0.21)$ & $0.81(0.37)$ & $0.68(0.20)$ & & \\
\hline (Z)-2-Nonen-1-ol & $4.80^{\mathrm{ab}}(1.53)$ & $6.52^{a}(1.95)$ & $4.17^{\mathrm{b}}(1.06)$ & KW & 0.041 \\
\hline 1-Nonanol & $0.05(0.05)$ & $0.24(0.24)$ & $0.24(0.16)$ & & \\
\hline \multicolumn{6}{|l|}{ Aldehydes } \\
\hline Pentanal & $8.04^{\mathrm{a}}(3.71)$ & $7.40^{\mathrm{ab}}(2.21)$ & $2.85^{\mathrm{b}}(1.23)$ & KW & 0.026 \\
\hline Hexanal & $283.7^{\mathrm{a}}(135.8)$ & $230.5^{\mathrm{a}}(123.7)$ & $44.7^{\mathrm{b}}(20.8)$ & KW; LDA & 0.001 \\
\hline Heptanal & $5.64^{\mathrm{a}}(2.44)$ & $9.90^{\mathrm{a}}(3.12)$ & $2.30^{b}(0.51)$ & KW; LDA & 0.002 \\
\hline Methional & $0.20(0.20)$ & $0.08(0.08)$ & $0.27(0.15)$ & & \\
\hline 2-Heptenal & $0.71^{\mathrm{a}}(0.42)$ & $0.54^{\mathrm{a}}(0.25)$ & $0.08^{b}(0.07)$ & KW; LDA & 0.001 \\
\hline Benzaldehyde & $3.07(1.31)$ & $2.71(0.57)$ & $3.14(0.84)$ & & \\
\hline Octanal & $16.0^{\mathrm{a}}(6.11)$ & $15.8^{\mathrm{a}}(6.35)$ & $6.18^{\mathrm{b}}(1.36)$ & KW; LDA & 0.004 \\
\hline 2-Octenal & $1.23^{\mathrm{b}}(0.68)$ & $3.02^{\mathrm{ab}}(1.22)$ & $3.50^{\mathrm{a}}(1.13)$ & KW & 0.045 \\
\hline Nonanal & $24.8^{\mathrm{a}}(8.06)$ & $31.6^{\mathrm{a}}(8.50)$ & $11.8^{\mathrm{b}}(2.52)$ & KW; LDA & 0.008 \\
\hline (E)-2-Nonenal & $0.53(0.26)$ & $0.54(0.13)$ & $0.33(0.05)$ & & \\
\hline (Z)-4-Decenal & $0.00(0.00)$ & $0.28(0.10)$ & $0.23(0.09)$ & & \\
\hline Decanal & $0.87^{\mathrm{a}}(0.25)$ & $0.96^{\mathrm{a}}(0.37)$ & $0.09^{\mathrm{b}}(0.09)$ & KW & 0.008 \\
\hline (E)-2-Decenal & $0.06^{\mathrm{ab}}(0.06)$ & $0.19^{\mathrm{a}}(0.07)$ & $0.00^{\mathrm{b}}(0.00)$ & KW; LDA & 0.003 \\
\hline$(E, E)-2,4$-Decadienal & $0.06(0.06)$ & $0.09(0.04)$ & $0.03(0.03)$ & & \\
\hline (E)-2-Undecenal & $0.00^{\mathrm{ab}}(0.00)$ & $0.07^{\mathrm{b}}(0.07)$ & $0.00^{\mathrm{a}}(0.00)$ & KW & 0.022 \\
\hline Dodecanal & $0.05(0.05)$ & $0.04(0.04)$ & $0.00(0.00)$ & & \\
\hline Tridecanal & nd & nd & nd & & \\
\hline Tetradecanal & nd & nd & nd & & \\
\hline \multicolumn{6}{|l|}{ Ketones } \\
\hline 2-Heptanone & $0.35(0.31)$ & $0.28(0.18)$ & $0.21(0.08)$ & & \\
\hline Butyrolactone & $0.42(0.40)$ & $0.97(0.72)$ & $0.24(0.16)$ & & \\
\hline 2-Methyl-6-heptanone & $0.00(0.00)$ & $0.00(0.00)$ & $0.00(0.00)$ & & \\
\hline 2-Nonanone & $0.00(0.00)$ & $0.00(0.00)$ & $0.00(0.00)$ & & \\
\hline \multicolumn{6}{|l|}{ Hydrocarbons } \\
\hline Toluene & $0.17^{\mathrm{a}}(0.17)$ & $0.03^{\mathrm{ab}}(0.03)$ & $0.00^{\mathrm{b}}(0.00)$ & KW & 0.048 \\
\hline 1,2,4-Trimethyl-cyclopentane & $0.68(0.68)$ & $0.32(0.23)$ & $0.16(0.16)$ & & \\
\hline Propyl-cyclohexane & $0.35(0.28)$ & $0.34(0.07)$ & $0.27(0.08)$ & & \\
\hline 4-Methyl-nonane & $0.00^{\mathrm{b}}(0.00)$ & $0.00^{\mathrm{b}}(0.00)$ & $0.22^{\mathrm{a}}(0.16)$ & KW; LDA & 0.002 \\
\hline
\end{tabular}


Table 2. Cont.

\begin{tabular}{|c|c|c|c|c|c|}
\hline & $\mathrm{C}^{1}$ & $\mathrm{SP}^{1}$ & $\mathrm{HI}^{1}$ & $\begin{array}{l}\text { Statistical } \\
\text { Analysis }\end{array}$ & $p$-Level \\
\hline 2,2,6-trimethyl-octane & $0.00^{\mathrm{a}}(0.00)$ & $0.00^{\mathrm{ab}}(0.00)$ & $0.48^{\mathrm{b}}(0.48)$ & KW; LDA & 0.004 \\
\hline 2,2,4,6-Pentamethyl-heptane & $53.5^{\mathrm{a}}(35.0)$ & $25.8^{\mathrm{a}}(4.86)$ & $19.3^{b}(6.90)$ & KW & 0.011 \\
\hline Decane & $4.24^{\mathrm{a}}(2.96)$ & $2.43^{\text {a }}(1.59)$ & $1.50^{\mathrm{b}}(0.44)$ & KW & 0.034 \\
\hline 2,2,4,4-Tetramethyl-octane & $4.34(1.86)$ & $1.24(1.24)$ & $0.73(0.73)$ & & \\
\hline 2,6,7-Trimethyl-decane & $1.43^{\mathrm{b}}(1.43)$ & $8.24^{\mathrm{a}}(2.20)$ & $10.8^{\mathrm{a}}(3.84)$ & KW; LDA & 0.000 \\
\hline 2-Methyl-decane & $0.00^{b}(0.00)$ & $2.42^{\mathrm{a}}(0.95)$ & $3.18^{\mathrm{a}}(1.27)$ & KW; LDA & 0.000 \\
\hline 5-Undecene & $0.29(0.29)$ & $2.37(1.33)$ & $2.50(1.09)$ & & \\
\hline Undecane & $1.57^{\mathrm{a}}(1.46)$ & $0.12^{\mathrm{b}}(0.08)$ & $0.09^{b}(0.04)$ & KW & 0.024 \\
\hline 2,8-dimethyl-4-methylene-nonane & $0.00^{b}(0.00)$ & $0.07^{\mathrm{ab}}(0.07)$ & $0.20^{\mathrm{a}}(0.19)$ & KW & 0.029 \\
\hline Pentyl-cyclohexane & $0.28(0.24)$ & $0.39(0.12)$ & $0.64(0.21)$ & & \\
\hline 3-Methylene-undecane & $0.25(0.23)$ & $0.26(0.14)$ & $0.39(0.14)$ & & \\
\hline Dodecane & $0.93(0.79)$ & $0.15(0.14)$ & $0.09(0.09)$ & & \\
\hline 2,6,11-trimethyl-dodecane & $0.05(0.05)$ & $0.03(0.03)$ & $0.00(0.00)$ & & \\
\hline Tridecane & $0.50(0.29)$ & $0.28(0.07)$ & $0.25(0.11)$ & & \\
\hline 2,3,5,8-tetramethyl-decane & $0.02(0.02)$ & $0.00(0.00)$ & $0.01(0.01)$ & & \\
\hline Tetradecane & $0.30(0.09)$ & $0.32(0.07)$ & $0.22(0.05)$ & & \\
\hline Pentadecane & $0.21(0.08)$ & $0.24(0.10)$ & $0.24(0.09)$ & & \\
\hline 2,6,10-trimethyl-tetradecane & $0.00(0.00)$ & $0.00(0.00)$ & $0.00(0.00)$ & & \\
\hline Hexadecane & $0.12(0.07)$ & $0.14(0.08)$ & $0.08(0.03)$ & & \\
\hline Heptadecane & $0.00(0.00)$ & $0.05(0.05)$ & $0.00(0.00)$ & & \\
\hline \multicolumn{6}{|l|}{ Thiols } \\
\hline 2-Ethyl-1-hexanethiol & $0.58^{\mathrm{b}}(0.58)$ & $5.86^{\mathrm{a}}(1.33)$ & $8.94^{\mathrm{a}}(3.24)$ & KW & 0.009 \\
\hline 2-Methyl-2-heptanethiol & $4.48^{\mathrm{b}}(1.00)$ & $7.29^{\mathrm{ab}}(1.64)$ & $9.68^{\mathrm{a}}(2.53)$ & KW & 0.009 \\
\hline \multicolumn{6}{|l|}{ Esters } \\
\hline $\begin{array}{c}\text { Pentanoic acid,2,2,4-trimethyl-3-hydroxy-, } \\
\text { isobutyl ester }\end{array}$ & $0.00(0.00)$ & $0.00(0.00)$ & $0.00(0.00)$ & & \\
\hline Carbamodithioic acid, diethyl-, methyl ester & nd & nd & nd & & \\
\hline Dimethyl phtalate & $1.85(0.53)$ & $1.93(0.95)$ & $1.35(0.41)$ & & \\
\hline $\begin{array}{l}\text { Pentanoic acid, } \\
\text { 2,2,4-trimethyl-3-carboxyisopropyl, } \\
\text { isobutyl ester }\end{array}$ & $0.04(0.04)$ & $0.10(0.06)$ & $0.07(0.04)$ & & \\
\hline \multicolumn{6}{|l|}{ Lactone } \\
\hline$\gamma$-Nonalactone & nd & nd & nd & & \\
\hline \multicolumn{6}{|l|}{ Acid } \\
\hline Dodecanoic acid & $0.00(0.00)$ & $0.00(0.00)$ & $0.00(0.00)$ & KW & 0.040 \\
\hline \multicolumn{6}{|l|}{ Nitrile } \\
\hline 4-Cyano-cyclohexene & $4.35^{\mathrm{a}}(2.31)$ & $0.98^{\mathrm{ab}}(0.98)$ & $0.31^{\mathrm{b}}(0.31)$ & KW & 0.041 \\
\hline \multicolumn{6}{|l|}{ Azide } \\
\hline 2-Azido-2,4,4,6,6-pentamethyl-heptane & nd & nd & nd & & \\
\hline \multicolumn{6}{|l|}{ Unknown } \\
\hline Unknown $(\mathrm{RT}=13.40 \mathrm{~min})$ & $0.35^{\mathrm{a}}(0.22)$ & $0.57^{\mathrm{a}}(0.33)$ & $0.00^{\mathrm{b}}(0.00)$ & KW; LDA & 0.006 \\
\hline Unknown ( $\mathrm{RT}=15.26 \mathrm{~min})$ & $1.78^{\mathrm{b}}(1.06)$ & $8.07^{\mathrm{a}}(2.91)$ & $10.2^{\mathrm{a}}(4.58)$ & KW; LDA & 0.000 \\
\hline Unknown $(\mathrm{RT}=16.51 \mathrm{~min})$ & $0.30^{\mathrm{a}}(0.30)$ & $0.00^{\mathrm{b}}(0.00)$ & $0.00^{\mathrm{ab}}(0.00)$ & KW & 0.020 \\
\hline Unknown $(\mathrm{RT}=18.15 \mathrm{~min})$ & $0.26^{\mathrm{ab}}(0.26)$ & $0.30^{\text {a }}(0.16)$ & $0.00^{\mathrm{b}}(0.00)$ & KW & 0.045 \\
\hline
\end{tabular}

\subsection{Volatile Compounds in Chicken Meat of Trial 1}

In total, 210 volatile compounds were detected in chicken breast meat produced with the three different diets in Trial 1. The results of the Kruskal-Wallis test, presented in Table 1, indicate that 16 compounds showed significant difference among the treatments, 6 of which were detected in trace amounts (i.e., $<0.30 \mathrm{ng} / \mathrm{g}$ ). The main groups of compounds affected by diet were alcohols (4), aldehydes (6), ketones (1), hydrocarbons (2), lactones (1), while 2 compounds were not identified (Retention time (RT): $17.96 \mathrm{~min}$ and RT = $28.62 \mathrm{~min}$ ). Two alcohols, 1-pentanol and 1-heptanol, were detected in lower levels in chicken breast meat produced with the HI diet compared to meat from the other two diets $(p<0.05)$, which did not differ from each other $(p>0.05)$. Levels of 1-hexanol, 1-octen-3-ol, hexanal 
and 2-heptenal, were lower under HI diet than under SP diet, neither of which differed from the C diet. In contrast, levels of 2-nonanone and pentadecane were higher in the HI diet compared to the other treatments, although levels were similar $(p>0.05)$ to the $\mathrm{C}$ diet and the SP diet, respectively.

Multivariate analysis was applied to investigate differences among the volatile profiles. LDA revealed that groups were clearly separated (Figure 1) with 18 compounds according to the confusion matrix and the kappa coefficient deriving from it. Four of these compounds were not identified (RTs: $17.96 \mathrm{~min} ; 23.86 \mathrm{~min} ; 28.62 \mathrm{~min}$ and $37.40 \mathrm{~min}$; Supplementary Table S1) and were detected in traces. The first component (explaining $74 \%$ of the variation) separated mainly the HI group (located on the left lower quadrant) from the SP group (right lower quadrant). The compounds that contributed mostly in the separation (i.e., factor loadings higher than 1.0) were 1-pentanol, hexanal, 1-hexanol, 2-heptenal, 1-heptanol, 1-octen-3-ol, 2-nonanone, 2,4-decadienal, $\gamma$-nonalactone, pentadecane, heptadecane. The second component (explaining 26\%) of the variation separated the C group (located on the upper side of the plot) from HI and SP group (bottom side of the plot). The compounds that contributed mostly to the separation (i.e., factor loadings higher than 1.0) were hexanal, 1-hexanol, 2-heptenal, 1-heptanol, 1-octen-3-ol, 2,4-decadienal and tridecanal (Supplementary Table S2).

\subsection{Volatile Compounds in Chicken Meat of Trial 2}

One hundred sixty-six volatile compounds were detected in the chicken breast meat of Trial 2. The results of the Kruskal-Wallis test (presented in Table 2) indicated that 33 compounds showed significant differences among treatments. The main groups of compounds that showed differences were alcohols (6), aldehydes (10), hydrocarbons (9), thiols (2), nitriles (1), and acids (1) while 4 compounds were not identified (unknown). In accordance with Trial 1, there were 7 compounds detected in trace amounts (i.e., $<0.30 \mathrm{ng} / \mathrm{g}$ ). Levels of 1-pentanol, 1-hexanol, 1-octen-3-ol, hexanal, heptanal, 2-heptenal, octanal, nonanal, decanal, 2,2,4,6-pentamethyl-heptane, decane, and one unknown (RT: $13.40 \mathrm{~min}$ ) were lower $(p<0.05)$ in chickens from the HI-dietary treatment compared to SP-fed and C-fed chicken. Levels of 4-ethyl-cyclohexanol, 1-octanol, 2-nonen-1-ol, pentanal, toluene, 2,2,6-trimethyl-octane, undecane, 4-cyano-cyclohexene, and one unknown (RT: 18.15) were lower $(p<0.05)$ in chickens fed the HI-diet than the ones from $C$ and/or the SP diet, which, in most cases, did not differ $(p>0.05)$. On the contrary, levels of 2-octenal, 2-undecenal, 4-methyl-nonane, 2,6,7-trimethyl-decane, 2-methyl-decane, 2,8-dimethyl-4-methylene-nonane, 2-ethyl-1-hexanethiol, 2-methyl-2-heptanethiol, 2-methyl-decane, and two unknown (RT:15.26 min and RT:16.51 min) were higher $(p<0.05)$ in HI-fed chickens in comparison to C-fed and/or the SP-fed chickens.

Linear discriminant analysis of the volatile data separated the dietary groups of Trial 2 with 14 compounds according to the kappa-coefficient (Figure 2). The first component (explaining 61.1\% of the variation) separated the HI group (left side of the plot) from the $C$ group (right side of the plot). The compounds that contributed mostly in the separation (i.e., factor loadings higher than 1.0) were hexanal, 1-hexanol, heptanal, 2-heptenal, 4-methyl-nonane, 2,2,6-trimethyl-octane, 1-octen-3-ol, 2,6,7-trimethyl-decane, and 2-decenal. The second component explained 39\% of the variation separated the SP group from the $\mathrm{HI}$ and $\mathrm{C}$ groups (Figure 2). The compounds that contributed to the separation were hexanal, 1-hexanol, heptanal, 2-heptenal, 4-methyl nonane, 2,2,6-trimethyl octane, 1-octen-3-ol, 2-ethyl-2-hexenol, 2,6,7-trimethyl-decane, 2-methyl-decane, and nonanal (Supplementary Table S3). 




Figure 1. Linear Discriminant Analysis plot of the volatile compounds of chicken meat under three dietary treatments in Trial 1. C diet: Soybean meal- based diet; SP diet: Soybean meal-based diet partially supplemented with Arthrospira platensis; HI diet: Soybean meal-based diet partially supplemented with Hermetia illucens larvae.

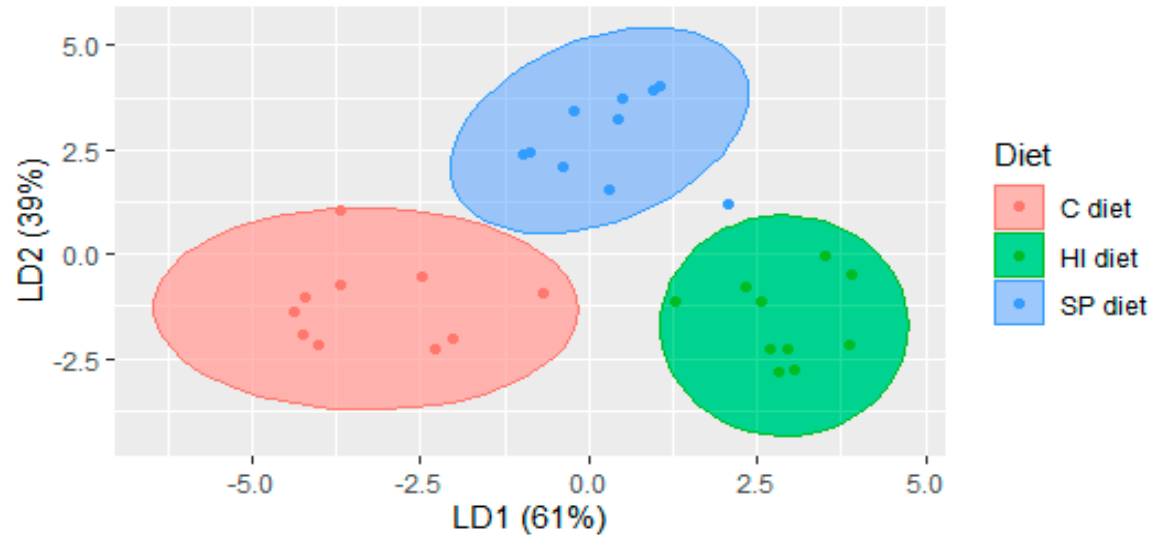

Figure 2. Linear discriminant analysis plot of the volatile compounds of chicken meat under three dietary treatments in Trial 2. C diet: Soybean meal-based diet; SP diet: Soybean meal-based diet supplemented with Arthrospira platensis; HI diet: Soybean meal-based diet supplemented with Hermetia illucens larvae.

\section{Discussion}

Several studies have investigated the effect of dietary treatment on chicken meat quality [20,38-40]. Results indicated that the volatile profile was affected by dietary treatments, with the majority of the compounds having been reported in literature on chicken meat aroma (Supplementary Table S1). Chicken meat is prone to lipid oxidation due to its high content of PUFAs; thus, volatile compounds deriving from lipid oxidation (i.e., aldehydes, alcohols, and ketones) [41] were expected.

Although a substantial number of compounds differed significantly among treatments of Trial 1 , only half of them were detected in considerable quantities (i.e., higher than $0.30 \mathrm{ng} / \mathrm{g}$ ). Results of Trial 2 indicated that the complete substitution of soybean meal with microalga or insects in the starter and grower phase strongly influenced the formation of lipid-derived compounds. Although acknowledging the fact that the two trials were independent and non-directly comparable, there was still a similar oxidation pattern observed among dietary treatments, i.e., the levels of compounds in meat produced with the HI-diet were usually lower than in the other dietary treatments, with most of the compounds deriving from linoleic acid (1-pentanol, 1-hexanol, 1-octen-3-ol, 2-nonen-1-ol, hexanal, heptanal, 2-heptenal) [42] or oleic acid (1-heptanol, 1-octanol, octanal, nonanal, decanal) [43].

The explanation of the results could be associated with the fatty acid (FA) profile of the diets since, in monogastric animals like chickens, the FA profile of the diet reflects the intramuscular FA composition (mainly the triglycerides, as the phospholipid composition is less affected [44]). 
The higher levels of the compounds in SP-fed and C-fed chicken compared to HI-fed chicken could be attributed to the possibly high level of PUFA in these diets [45-48], which could have promoted lipid oxidation. Cortinas Hernández et al. [38] attributed the linear increase of lipid oxidation in cooked chicken meat to the increasing PUFA content in raw meat. In this regard, Bonos, et al. [49] reported that dietary supplementation with 5 or $10 \mathrm{~g}$ spirulina per $\mathrm{kg}$ feed influenced the fatty acid composition of thigh muscle (by enhancing the PUFA content), but not of the breast muscle which had similar PUFA, saturated fatty acid (SFA), and mono unsaturated fatty acid (MUFA) content with the control diet. In contrast, El-Bahr et al. [24] reported that inclusion of $1 \mathrm{~g}$ spirulina per kg feed in a corn-soybean basal diet increased the major long chain n-3 PUFA (eicosapentaenoic, docosahexaenoic, and arachidonic acid) and total PUFA content in breast muscle, while the SFA, MUFA, and PUFA/SFA ratio remained unchanged.

In the case of an insect-based diet, the FA composition of chicken meat depends on the fatty acid profile of the insect lipids, which in turn may be affected by FA composition of the rearing substrate [14] or the stage in the life cycle of the insect [50]. HI larvae has been associated with high SFA content (ranging usually between $40-80 \%$ ) and low PUFA content, i.e., around $10-20 \%$ of total FA [51] or even less, in larvae [52,53]. Thus, the lower extent of oxidation observed in muscle from HI-fed chicken could be associated with the reduced content of the easily oxidized PUFA and the elevated content of the (slowly oxidized) SFA in HI treatment. In support of this, Schiavone et al. [18] reported that $50 \%$ or $100 \%$ substitution of soybean oil with HI larvae increased the ratio of SFA of broiler chicken breast proportionally to the level of substitution and to the detriment of the PUFA fraction. The diminishing effect of HI larvae (meal or fat) on PUFA content was confirmed in Schiavone et al. [53] and Cullere et al. [54]. In addition, Schiavone et al. [53] noted that the defatting process not only enhances the protein content but may also reduce the risk of lipid oxidation in meat. Finally, volatile analysis showed that hexanal, a compound considered as an oxidation marker [55], was detected in lower levels under the HI diet than under the other two diets in both trials, which could imply less oxidation. Although the fatty acid profile of breast meat in our studies was not analyzed, a recent study by Altmann et al. [20] using the same animals as in Trial 1 reported a higher content of SFA in thigh muscle from chickens fed with the HI diet. It should be noted that lipid content between breast and thigh muscle in poultry meat differs [56] as well as the fatty acid composition [57]. As a consequence, the results of Altmann et al. [20] can only be seen as indicative.

On the other hand, the higher levels of the two thiols in HI-fed chickens compared with the other two diets in Trial 2 could reflect differences in the free amino acid content among the three treatments, especially considering that thiol formation is linked with Strecker degradation of amino acids (e.g., cysteine) when reacting with secondary lipid oxidation compounds [58]. Differences in thiamine content may also play a role, as thiamine degradation is another pathway for thiol formation [59].

It appears that several factors could be involved in the extent of lipid oxidation when feeds of high nutritional value, like microalga or insects, are included in poultry diets. These factors may be related to the type of microalga [24] or insect species [60], the level of supplementation, the stage of inclusion in the basal diets (starter, grower, finisher phase), the type and level of antioxidants (e.g., $\beta$-carotene, tocopherol, carotenoids) that microalga contain and may affect the oxidative stability [61], the type of substrate on which larvae were reared [14], the use of defatted biomasses (algae or insect) [62,63], the defatting method [64], or the interaction with other factors, like amino acid supplementation [24,65].

Overall, the results of the two trials indicate that alternative protein sources may affect the aromatic profile of chicken meat and this could impact sensory perception. For example, hexanal (detected in the highest abundance in both trials) is associated with a "green/grassy" odor [66] and has been identified as one of the most important odor active compounds in chicken breast on the basis of its odor activity value [67]. 2-Heptenal, heptanal, octanal, nonanal, and decanal (described as "oily/fatty", "fatty/roasty/citrus", "fatty/sweet", "roasted/meaty/fatty", and "sweet/fruity", respectively $[66,68]$ ) due to their low odor threshold values [69] and the relatively high level at which they were detected 
in C and SP diets, mainly in Trial 2, could potentially affect aroma or flavor perception in chickens fed these diets. Although the significant alcohols have been previously reported in chicken aroma studies, only 1-octen-3-ol, with the characteristic "mushroom" odor [68], has a low odor threshold ( $1 \mathrm{ng} / \mathrm{g}$ ) compared to the other alcohols $[69,70]$ and was detected in significant quantities in both trials. Hydrocarbons are derived from lipid oxidation and are compounds that showed significant variations among treatments. However, these are generally not considered to impact the flavor of lipid-based foods [71]. Thiols are significant volatile compounds in meat aroma [72]. The higher levels of the two thiols in HI-fed chicken compared with the other two diets in Trial 2 could signify differences in the aromatic quality of the three diets. As meat flavor is a combination of volatile aroma and non-volatile taste compounds, further analytical research (e.g., defining free amino acid content; performing gas chromatography-olfactometry analysis) in combination with sensory evaluation would be required to identify compounds that could influence eating quality and possibly consumer preference.

LDA indicated the potential of the volatile analysis/profile to discriminate the three dietary treatments in both trials. Compounds that had higher impact on the group separation were mainly those with higher concentration, rather than compounds that were only detected in traces. The content of linoleic acid-the precursor of most of these compounds—seemed to play a significant role in the characterization and identification of these treatments.

\section{Conclusions}

This is the first study to report differences in the volatile profile of chicken meat after dietary replacement of soybean meal with alternative protein sources (i.e., microalga or partially defatted larvae meal). The differences detected were mainly in the lipid oxidation-deriving compounds that could play a significant role in the development of the typical aroma of chicken meat. Multivariate analysis confirmed that the dietary treatments led to a discriminatory volatile profile in both trials. Considering that both microalga and insects could stand as sustainable options in animal feeding in the years to come, future research should focus on identifying the type of biomasses and the proper inclusion level in order to improve chicken meat quality.

Supplementary Materials: The following are available online at http://www.mdpi.com/2304-8158/9/9/1235/s1, Table S1: Linear retention indices (LRIs) and method of identification of the main compounds detected in chicken meat (Trial 1 and Trial 2); Table S2: Factor loadings of the 18 most discriminant compounds of Trial 1 that lead to a confusion matrix with a Cohen's kappa coefficient value of 1.0, i.e., clear separation of the three groups, Table S3: Factor loadings of the 14 most discriminant compounds of Trial 2 that lead to a confusion matrix with a Cohen's kappa coefficient value of 1.0, i.e., clear separation of the three groups.

Author Contributions: Conceptualization, B.A.A. and D.M.; methodology, V.G.; statistical analysis, A.O.S. and V.G.; validation, V.G. and M.C.; formal analysis, V.G.; investigation, V.G.; resources, D.M.; data curation, V.G.; writing—original draft preparation, V.G.; writing—review and editing, V.G.; M.C.; B.A.A., A.O.S., and D.M.; visualization, V.G., M.C., B.A.A. and D.M.; supervision, V.G., M.C., and D.M.; project administration, B.A.A. and D.M.; funding acquisition, D.M. All authors have read and agreed to the published version of the manuscript.

Funding: This research was funded by the initiative Niedersächsisches Vorab by the Ministry for Science and Culture, Lower Saxony, Germany (\#ZN 3041).

Acknowledgments: We would like to thank Carmen Neumann, Susanne Rothstein, and Frank Liebert from the Division Animal Nutrition Physiology, University of Göttingen, Germany, for their contributions towards the animal nutrition and animal rearing. We also would like to thank Ruth Wigger for technical maintenance of the GC-MS and Elisa Oertel for assisting in data preprocessing (peak alignment) in the two trials. This study would not be possible without the many technical staff at the Department of Animal Sciences, University of Göttingen, who provided support during animal slaughtering.

Conflicts of Interest: The authors declare no conflict of interest.

\section{References}

1. Phillips, S.M.; Chevalier, S.; Leidy, H.J. Protein "requirements" beyond the RDA: Implications for optimizing health. Appl. Physiol. Nutr. Metab. 2016, 41, 565-572. [CrossRef] [PubMed] 
2. Kralik, G.; Kralik, Z.; Grčević, M.; Hanžek, D. Quality of Chicken Meat. Anim. Husb. Nutr. $2018,63$. [CrossRef]

3. Mir, N.A.; Rafiq, A.; Kumar, F.; Singh, V.; Shukla, V. Determinants of broiler chicken meat quality and factors affecting them: A review. J. Food Sci. Technol. 2017, 54, 2997-3009. [CrossRef]

4. Bou, R.; Guardiola, F.; Barroeta, A.; Codony, R. Effect of dietary fat sources and zinc and selenium supplements on the composition and consumer acceptability of chicken meat. Poult. Sci. 2005, 84, 1129-1140. [CrossRef] [PubMed]

5. Kareem, K.Y.; Abdulla, N.R.; Foo, H.L.; Zamri, A.N.M.; Shazali, N.; Loh, T.C.; Alshelmani, M.I. Effect of feeding larvae meal in the diets on growth performance, nutrient digestibility and meat quality in broiler chicken. Indian J. Anim. Res. 2018, 88, 1180-1185.

6. Gaylord, T.; Barrows, F.; Rawles, S. Apparent amino acid availability from feedstuffs in extruded diets for rainbow trout Oncorhynchus mykiss. Aquac. Nutr. 2010, 16, 400-406. [CrossRef]

7. Hanson, M.N.; Persia, M.E. Effects of Dietary Soy Inclusion on Broiler Chick Performance and Metabolizable Energy. Iowa State Univ. Anim. Ind. Rep. 2014. [CrossRef]

8. Veldkamp, T.; van Duinkerken, G.; van Huis, A.; Lakemond, C.M.M.; Bosch, G.; van Boekel, M.A.J.S. Insects as a Sustainable Feed Ingredient in Pig and Poultry Diets-A Feasibility Study; Wageningen UR Livestock Research: Lelystad, The Netherlands, 2012.

9. OECD/FAO. OECD-FAO Agricultural Outlook 2020-2029; OECD: Paris, France, 2020. [CrossRef]

10. Kristensen, L.; Støier, S.; Würtz, J.; Hinrichsen, L. Trends in meat science and technology: The future looks bright, but the journey will be long. Meat Sci. 2014, 98, 322-329. [CrossRef]

11. Finley, J.W. Evolution and future needs of food chemistry in a changing world. J. Agric. Food Chem. 2020, in press. [CrossRef]

12. Akhtar, Y.; Isman, M.B. Insects as an Alternative Protein Source. In Proteins in Food Processing, 2nd ed.; Yada, Y.R., Ed.; Woodhead Publishing: Cambridge, UK, 2017; pp. 263-288.

13. Madeira, M.S.; Cardoso, C.; Lopes, P.A.; Coelho, D.; Afonso, C.; Bandarra, N.M.; Prates, J.A.M. Microalgae as feed ingredients for livestock production and meat quality: A review. Livest. Sci. 2017, 205, 111-121. [CrossRef]

14. Barragan-Fonseca, K.B.; Dicke, M.; van Loon, J.J. Nutritional value of the black soldier fly (Hermetia illucens L.) and its suitability as animal feed-A review. J. Insects Food Feed. 2017, 3, 105-120. [CrossRef]

15. Józefiak, D.; Józefiak, A.; Kierończyk, B.; Rawski, M.; Świątkiewicz, S.; Długosz, J.; Engberg, R.M. Insects-A Natural Nutrient Source for Poultry-A Review. Ann. Anim. Sci. 2016, 16, 297-313. [CrossRef]

16. Müller, A.; Wolf, D.; Gutzeit, H.O. The black soldier fly, Hermetia illucens-A promising source for sustainable production of proteins, lipids and bioactive substances. Z. Naturforsch. C Biosci. 2017, 72, 351-363. [CrossRef] [PubMed]

17. Gasco, L.; Biasato, I.; Dabbou, S.; Schiavone, A.; Gai, F. Animals fed insect-based diets: State-of-the-art on digestibility, performance and product quality. Animals 2019, 9, 170. [CrossRef]

18. Schiavone, A.; Cullere, M.; De Marco, M.; Meneguz, M.; Biasato, I.; Bergagna, S.; Dezzutto, D.; Gai, F.; Dabbou, S.; Gasco, L. Partial or total replacement of soybean oil by black soldier fly larvae (Hermetia illucens L.) fat in broiler diets: Effect on growth performances, feed-choice, blood traits, carcass characteristics and meat quality. Ital. J. Anim. Sci. 2017, 16, 93-100. [CrossRef]

19. Cullere, M.; Tasoniero, G.; Giaccone, V.; Acuti, G.; Marangon, A.; Dalle Zotte, A. Black soldier fly as dietary protein source for broiler quails: Meat proximate composition, fatty acid and amino acid profile, oxidative status and sensory traits. Animals 2018, 12, 640-647. [CrossRef]

20. Altmann, B.; Wigger, R.; Ciulu, M.; Mörlein, D. The effect of insect or microalga alternative protein feeds on broiler meat quality. J. Sci. Food Agric. 2020. [CrossRef]

21. Falquet, J.; Hurni, J.P. The Nutritional Aspects of Spirulina; Antenna Foundation: Geneva, Switzerland, 2017.

22. Jung, F.; Krüger-Genge, A.; Waldeck, P.; Küpper, J.-H. Spirulina platensis, a super food? J. Cell. Biotechnol. 2019, 5, 43-54. [CrossRef]

23. Khan, Z.; Bhadouria, P.; Bisen, P. Nutritional and therapeutic potential of Spirulina. Curr. Pharm. Biotechnol. 2005, 6, 373-379. [CrossRef]

24. El-Bahr, S.; Shousha, S.; Shehab, A.; Khattab, W.; Ahmed-Farid, O.; Sabike, I.; El-Garhy, O.; Albokhadaim, I.; Albosadah, K. Effect of Dietary Microalgae on Growth Performance, Profiles of Amino and Fatty Acids, Antioxidant Status, and Meat Quality of Broiler Chickens. Animals 2020, 10, 761. [CrossRef] 
25. El-Hady, A.A.; El-Ghalid, O. Spirulina platensis Algae (SPA): A novel poultry feed additive Effect of SPA supplementation in broiler chicken diets on productive performance, lipid profile and calcium-phosphorus metabolism. In Proceedings of the VI Mediterranean Poultry Summit, Torino, Italy, 18-20 June 2018.

26. Venkataraman, L.V.; Somasekaran, T.; Becker, E.W. Replacement Value of blue-green alga (spirulina platensis) for fishmeal and a vitamin-mineral premix for broiler chicks. Br. Poult. Sci. 1994, 35, 373-381. [CrossRef] [PubMed]

27. Vasta, V.; Priolo, A. Ruminant fat volatiles as affected by diet. A review. Meat Sci. 2006, 73, $218-228$. [CrossRef]

28. Calkins, C.R.; Hodgen, J.M. A fresh look at meat flavor. Meat Sci. 2007, 77, 63-80. [CrossRef] [PubMed]

29. Flores, M. The Eating Quality of Meat: III-Flavor. In Lawrie's Meat Science, 8th ed.; Toldrá, F., Ed.; Woodhead Publishing Series in Food Science; Technology and Nutrition: Cambridge, UK, 2017; pp. 383-417. [CrossRef]

30. Monahan, F.J.; Schmidt, O.; Moloney, A.P. Meat provenance: Authentication of geographical origin and dietary background of meat. Meat Sci. 2018, 144, 2-14. [CrossRef] [PubMed]

31. Neumann, C.; Velten, S.; Liebert, F. The Graded Inclusion of Algae (Spirulina platensis) or Insect (Hermetia illucens) Meal as a Soybean Meal Substitute in Meat Type Chicken Diets Impacts on Growth, Nutrient Deposition and Dietary Protein Quality Depending on the Extent of Amino Acid Supplementation. Open J. Anim. Sci. 2018, 8, 163-183. [CrossRef]

32. Wang, C.; Zhang, W.; Li, H.; Mao, J.; Guo, C.; Ding, R.; Wang, Y.; Fang, L.; Chen, Z.; Yang, G. Analysis of volatile compounds in pears by HS-SPME-GC $\times$ GC-TOFMS. Molecules 2019, 24, 1795. [CrossRef]

33. R Core Team. R A Language and Environment for Statistical Computing; R Foundation for Statistical Computing: Vienna, Austria, 2019.

34. Ottensmann, M.; Stoffel, M.A.; Nichols, H.J.; Hoffman, J.I. GCalignR: An R package for aligning gas-chromatography data for ecological and evolutionary studies. PLoS ONE 2018, 13. [CrossRef]

35. Robinson, D. Fuzzyjoin: Join Tables Together on Inexact Matching. R Package Version 0.1.6. 2020. Available online: https://cran.r-project.org/web/packages/fuzzyjoin/index.html (accessed on 4 September 2020).

36. Venables, W.N.; Ripley, B.D. Modern Applied Statistics with S, 4th ed.; Springer: New York, NY, USA, 2002.

37. Revelle, W. Procedures for Psychological, Psychometric, and Personality Research; American Chemical Society: Washington, DC, USA, 2019.

38. Cortinas Hernández, L.; Barroeta, A.C.; Villaverde Haro, C.; Galobarti Cots, J.; Guardiola Ibarz, F.; Baucells Sánchez, M.D. Influence of the Dietary Polyunsaturation Level on Chicken Meat Quality: Lipid Oxidation. Poult. Sci. 2005, 84, 48-55. [CrossRef]

39. Cullere, M.; Woods, M.J.; van Emmenes, L.; Pieterse, E.; Hoffman, L.C.; Dalle Zotte, A. Hermetia illucens Larvae Reared on Different Substrates in Broiler Quail Diets: Effect on Physicochemical and Sensory Quality of the Quail Meat. Animals 2019, 9, 525. [CrossRef]

40. Lopez-Ferrer, S.; Baucells, M.D.; Barroeta, A.; Grashorn, M. n-3 enrichment of chicken meat. 1. Use of very long-chain fatty acids in chicken diets and their influence on meat quality: Fish oil. Poult. Sci. 2001, 80, 741-752. [CrossRef]

41. Jayasena, D.D.; Ahn, D.U.; Nam, K.C.; Jo, C. Flavour Chemistry of Chicken Meat: A Review. Asian-Australas J. Anim. Sci. 2013, 26, 732-742. [CrossRef] [PubMed]

42. Elmore, J.S.; Cooper, S.L.; Enser, M.; Mottram, D.S.; Sinclair, L.A.; Wilkinson, R.G.; Wood, J.D. Dietary manipulation of fatty acid composition in lamb meat and its effect on the volatile aroma compounds of grilled lamb. Meat Sci. 2005, 69, 233-242. [CrossRef]

43. Vandamme, J.; Nikiforov, A.; De Roose, M.; Leys, C.; De Cooman, L.; Van Durme, J. Controlled accelerated oxidation of oleic acid using a DBD plasma: Determination of volatile oxidation compounds. Food Res. Int. 2016, 79, 54-63. [CrossRef]

44. Raes, K.; De Smet, S.; Demeyer, D. Effect of dietary fatty acids on incorporation of long chain polyunsaturated fatty acids and conjugated linoleic acid in lamb, beef and pork meat: A review. Anim. Feed. Sci. Technol. 2004, 113, 199-221. [CrossRef]

45. Diraman, H.; Koru, E.; Dibeklioglu, H. Fatty acid profile of Spirulina platensis used as a food supplement. Isr. J. Aquacult. Bamidgeh 2009, 91, 134-142.

46. Hudson, B.J.; Karis, I.G. The lipids of the alga Spirulina. J. Sci. Food Agric. 1974, 25, 759-763. [CrossRef] [PubMed] 
47. Azman, M.; Konar, V.; Seven, P. Effects of different dietary fat sources on growth performances and carcass fatty acid composition of broiler chickens. Rev. Med. Vet. 2004, 155, 278-286.

48. Waldroup, P.; Waldroup, A. Fatty acid effect on carcass: The influence of various blends of dietary fats added to corn-soybean meal based diets on the fatty acid composition of broilers. Int. J. Poult. Sci. 2005, 4, 123-132. [CrossRef]

49. Bonos, E.; Kasapidou, E.; Kargopoulos, A.; Karampampas, A.; Christaki, E.; Florou-Paneri, P.; Nikolakakis, I. Spirulina as a functional ingredient in broiler chicken diets. S. Afr. J. Anim. Sci. 2016, 46, 94. [CrossRef]

50. Hadžić, D. Using of Black Soldier Fly (Hermetia Illucens) Larvae Meal in Fish Nutrition. In Answers for Forthcoming Challenges in Modern Agriculture; Brka, M.O.-M.E., Karić, L., Falan, V., Toroman, A., Eds.; Springer: Cham, Switzerland, 2020; pp. 132-140.

51. Ewald, N. Fatty Acid Composition of Black Soldier Fly Larvae. Master's Thesis, Swedish University of Agricultural Sciences, Uppsala, Sweden, 2019.

52. Ushakova, N.; Brodskii, E.; Kovalenko, A.; Bastrakov, A.; Kozlova, A.; Pavlov, D. Characteristics of lipid fractions of larvae of the black soldier fly Hermetia illucens. Dokl. Biochem. Biophys. 2016, 468, 209-212. [CrossRef]

53. Schiavone, A.; Dabbou, S.; Petracci, M.; Zampiga, M.; Sirri, F.; Biasato, I.; Gai, F.; Gasco, L. Black soldier fly defatted meal as a dietary protein source for broiler chickens: Effects on carcass traits, breast meat quality and safety. Animal 2019, 13, 2397-2405. [CrossRef] [PubMed]

54. Cullere, M.; Schiavone, A.; Dabbou, S.; Gasco, L.; Dalle Zotte, A. Meat quality and sensory traits of finisher broiler chickens fed with black soldier fly (Hermetia Illucens L.) larvae fat as alternative fat source. Animals 2019, 9, 140. [CrossRef] [PubMed]

55. Shahidi, F.; Pegg, R.B. Hexanal as an indicator of the flavor deterioration of meat and meat products. In Lipids in Food Flavours; Ho, C.T., Hartman, T.G., Eds.; ACS Publications: Denver, CO, USA, 1994; pp. 256-279.

56. Barroeta, A. Nutritive value of poultry meat: Relationship between vitamin E and PUFA. World Poult. Sci. J. 2007, 63, 277-284. [CrossRef]

57. Schneiderová, D.; Zelenka, J.; Mrkvicová, E. Poultry meat production as a functional food with a voluntary n-6 and n-3 polyunsaturated fatty acids ratio. Czech. J. Anim. Sci. 2007, 52, 203-213. [CrossRef]

58. Whitfield, F.B.; Mottram, D.S. Volatiles from interactions of Maillard reactions and lipids. Crit. Rev. Food Sci. Nutr. 1992, 31, 1-58. [CrossRef]

59. Belitz, H.D.; Grosch, W.; Schieberle, P. 5. Aroma Substances. In Food Chemistry; Springer: Berlin/Heidelberg, Germany, 2004; pp. 319-376.

60. Vrabec, V.; Kulma, M.; Cocan, D. Insects as an Alternative Protein Source for Animal Feeding: A Short Review about Chemical Composition. Bul. Univ. Agric. Sci. Vet. Med. Cluj-Napoca Anim. Sci. Biotechnol. 2014, 72, 116-126. [CrossRef]

61. Pestana, J.; Puerta, B.; Santos, H.; Madeira, M.; Alfaia, C.; Lopes, P.; Pinto, R.; Lemos, J.; Fontes, C.; Lordelo, M. Impact of dietary incorporation of Spirulina (Arthrospira platensis) and exogenous enzymes on broiler performance, carcass traits, and meat quality. Poult. Sci. 2020, 99, 2519-2532. [CrossRef]

62. Schiavone, A.; De Marco, M.; Martínez, S.; Dabbou, S.; Renna, M.; Madrid, J.; Hernandez, F.; Rotolo, L.; Costa, P.; Gai, F. Nutritional value of a partially defatted and a highly defatted black soldier fly larvae (Hermetia illucens L.) meal for broiler chickens: Apparent nutrient digestibility, apparent metabolizable energy and apparent ileal amino acid digestibility. J. Anim. Sci. Biotech. 2017, 8, 51. [CrossRef]

63. Gatrell, S.K.; Kim, J.; Derksen, T.J.; O’Neil, E.V.; Lei, X.G. Creating $\omega-3$ fatty-acid-enriched chicken using defatted green microalgal biomass. J. Agric. Food Chem. 2015, 63, 9315-9322. [CrossRef]

64. Laroche, M.; Perreault, V.; Marciniak, A.; Gravel, A.; Chamberland, J.; Doyen, A. Comparison of Conventional and Sustainable Lipid Extraction Methods for the Production of Oil and Protein Isolate from Edible Insect Meal. Foods 2019, 8, 572. [CrossRef]

65. Moghadam, M.B.; Shehab, A.; Cherian, G. Methionine supplementation augments tissue n-3 fatty acid and tocopherol content in broiler birds fed flaxseed. Anim. Feed. Sci. Technol. 2017, 228, 149-158. [CrossRef]

66. Zhang, M.; Chen, X.; Hayat, K.; Duhoranimana, E.; Zhang, X.; Xia, S.; Yu, J.; Xing, F. Characterization of odor-active compounds of chicken broth and improved flavor by thermal modulation in electrical stewpots. Food Res. Int. 2018, 109, 72-81. [CrossRef] [PubMed]

67. Ayseli, M.T.; Filik, G.; Selli, S. Evaluation of volatile compounds in chicken breast meat using simultaneous distillation and extraction with odour activity value. J. Food Nutr. Res. 2014, 53, 137-142. 
68. Ba, H.V.; Hwang, I.; Jeong, D.; Touseef, A. Principle of Meat Aroma Flavors and Future Prospect. In Latest Research into Quality Control; Akyar, I., Ed.; InTech: Rijeka, Croatia, 2012; pp. 146-176.

69. Qi, J.; Wang, H.; Zhou, G.; Xu, X.; Li, X.; Bai, Y.; Yu, X. Evaluation of the taste-active and volatile compounds in stewed meat from the Chinese yellow-feather chicken breed. Int. J. Food Prop. 2018, 20, S2579-S2595. [CrossRef]

70. Gkarane, V.; Brunton, N.P.; Harrison, S.M.; Gravador, R.S.; Allen, P.; Claffey, N.A.; Diskin, M.G.; Fahey, A.G.; Farmer, L.J.; Moloney, A.P. Volatile Profile of Grilled Lamb as Affected by Castration and Age at Slaughter in Two Breeds. J. Food Sci. 2018, 83, 2466-2477. [CrossRef] [PubMed]

71. Ho, C.-T.; Chen, Q. Lipids in food flavors. An overview. In Lipids in Food Flavors; Ho, C.-T., Hartman, T.G., Eds.; American Chemical Society: Washington, DC, USA, 1994; pp. 1-14.

72. Mottram, D.S. Flavour formation in meat and meat products: A review. Food Chem. 1998, 62, 415-424. [CrossRef]

(C) 2020 by the authors. Licensee MDPI, Basel, Switzerland. This article is an open access article distributed under the terms and conditions of the Creative Commons Attribution (CC BY) license (http://creativecommons.org/licenses/by/4.0/). 